\title{
ANÁLISIS DE LOS VALORES CÍVICOS EN LOS MANUALES DE TEORÍA E HISTORIA DE LA EDUCACIÓN PARA LA FORMACIÓN DE MAESTROS EN ESPAÑA
}

ANALYSIS OF CIVIC VALUES IN THE TEXTBOOKS OF THEORY AND HISTORY OF

EDUCATION FOR TRAINING OF TEACHERS IN SPAIN

\section{Volumen 15, Número 2}

Mayo - Agosto

pp. 1-23

Eva Ortiz Cermeño

Tomás Izquierdo Rus

Pedro Miralles Martínez

\section{Revista indizada en REDALYC, SCIELO}

Revista distribuida en las bases de datos:

CATÁLOGO DE LATINDEX, IRESIE, CLASE, DIALNET, DOAJ, E-REVIST@S, SHERPA/ROMEO, QUALIS, MIAR

Revista registrada en los directorios:

ULRICH'S, REDIE, RINACE, OEI, MAESTROTECA, PREAL, $\underline{\text { CLACSO }}$ 


\title{
ANÁLISIS DE LOS VALORES CÍVICOS EN LOS MANUALES DE TEORÍA E HISTORIA DE LA EDUCACIÓN PARA LA FORMACIÓN DE MAESTROS EN ESPAÑA
}

\author{
ANALYSIS OF CIVIC VALUES IN THE TEXTBOOKS OF THEORY AND HISTORY OF \\ EDUCATION FOR TRAINING OF TEACHERS IN SPAIN
}

\author{
Eva Ortiz Cermeño ${ }^{1}$ \\ Tomás Izquierdo Rus ${ }^{2}$ \\ Pedro Miralles Martínez ${ }^{3}$
}

\begin{abstract}
Resumen: Este trabajo analiza los valores cívicos (libertad, disciplina, responsabilidad, participación, cooperación, etc.) presentes en los manuales universitarios, en la asignatura Teorías e Instituciones Contemporáneas de Educación, materia que se imparte, en el grado de Maestro de Educación Primaria, en las universidades españolas. Se ha utilizado una metodología cualitativa de tipo descriptivo e inductivo para explicar los datos obtenidos. Se lleva a cabo con la ayuda del programa informático ATLAS.ti para elaborar diagramas de redes y así, poder categorizar y sintetizar los datos analizados. Los resultados obtenidos indican que los autores estudiados inciden en la libertad y la disciplina para potenciar el pensamiento crítico de los estudiantes. Se concluye que existe una relación positiva entre los valores analizados y las teorías educativas por su relevancia en la actualidad.
\end{abstract}

Palabras clave: VALORES, CIUDADANÍA, FORMACIÓN DE MAESTROS, MANUALES UNIVERSITARIOS, ESPAÑA

Abstract: This work analyses the civic values (freedom, discipline, responsibility, participation, cooperation, etc.) in university textbooks on the Theories and Contemporary Institutions of Education, subject taught in the degree of Master of Primary Education in Spanish universities. It has been used a descriptive and inductive type qualitative methodology to explain the data. Is carried out with the help of computer program ATLAS.ti. to develop network diagrams and categorize and synthesize the data analyzed. The results indicate that the authors studied the impact on freedom and discipline to enhance students critical thinking. We conclude that there is a positive relationship between the analyzed values and educational theories by their relevance today.

Key words: VALUES, CITIZENSHIP, TEACHER EDUCATION, TEXTBOOKS, SPAIN

\footnotetext{
${ }^{1}$ Profesora Asociada de Didáctica de las Ciencias Sociales de la Universidad de Murcia, España y Orientadora Pedagógica. Doctoranda en Pedagogía. Dirección electrónica: evaortiz@um.es

2 Profesor Contratado Doctor del Departamento de Métodos de Investigación y Diagnóstico en Educación de la Universidad de Murcia, España. Doctor por la Universidad de Granada.Dirección electrónica: tomasiza@um.es

${ }^{3}$ Doctor, Profesor Titular de Didáctica de las Ciencias Sociales y Vicerrector de Formación e Innovación de la Universidad de Murcia, España. Dirección electrónica:pedromir@um.es
}

Artículo recibido: 23 de agosto, 2014

Enviado a corrección: 4 de diciembre, 2014

Aprobado: 23 de febrero, 2015 


\section{Introducción}

La educación en valores constituye uno de los principales retos en el ámbito de la educación. Las reformas educativas de nuestro entorno cultural han contemplado el concepto de temas o ejes transversales como elementos curriculares que se introducen en todo el proceso educativo para desarrollar determinados valores. Como comentan Paredes y Ribera (2006) resulta crucial coordinar y tratar de manera conjunta los valores desarrollados a través del currículo para que no queden descontextualizados, porque estos son un marco de referencia personal que orienta a las personas en sus acciones, opiniones y decisiones.

Los valores se construyen a partir de la relación de cada individuo con su entorno cultural y familiar, sus experiencias de vida y su propia capacidad de análisis. La educación en valores consiste en preparar para aprender a convivir y a profundizar en valores éticos y democráticos esenciales en la sociedad actual. Estos no se enseñan, se aprenden. Así pues, desde una perspectiva constructivista del aprendizaje se trata de facilitar momentos que permitan cultivar la autonomía personal, la voluntad, el respeto a la diferencia, la aceptación de contrariedades y la disponibilidad al diálogo como una virtud y como un método para afrontar las relaciones con los demás.

La selección de unos valores y no de otros es siempre arriesgada y, más todavía, cuando incide en un elemento tan trascendental como la educación. Escoger es renunciar y renunciar es siempre comprometido. De todas maneras, según manifiestan Pujol y Luz (2003), los valores humanos se entrelazan entre ellos y resulta difícil distinguir dónde termina uno y dónde empieza otro. Dicho de otro modo, no es factible discernir si se está educando en el diálogo o en la paz o en la justicia, porque cabe preguntarse: ¿Es posible una paz sin diálogo? ¿Podría existir la paz al margen de la justicia? ¿La urbanidad no es un aspecto del respeto? ¿Es concebible la amistad sin la sinceridad? y ¿una responsabilidad carente de prudencia? Por ello, no se puede ser más tolerantes sin ser a la vez más generosos, más comprensivos, más dialogantes, más respetuosos, etc. Así pues:

Aceptar los valores vigentes (conservarlos y aumentarlos), o bien cambiar la situación dada por antivaliosa es siempre el problema y el quehacer de toda educación. Adaptación o cambio, aceptación o rechazo es quizás, hoy más que nunca, una tarea permanente dada la rapidez de los cambios derivada del análisis y valoración de lo heredado. (Gervilla, 1992, p. 432) 
Los valores son cualidades que las personas y los pueblos, a través de la historia y en la actualidad, descubrimos para hacer nuestro mundo más habitable, más humano. La reflexión ética de nuestro tiempo ha puesto de manifiesto que la dignidad de la persona, la libertad, la justicia, la igualdad, la solidaridad, el pluralismo político o la democracia, el civismo, la tolerancia, la participación, la paz y la responsabilidad son los anclajes de la ciudadanía moderna. Cifuentes (2008) sostiene que éstos deben basarse en los derechos humanos. Según Escámez (2002, p. 26): "son los mínimos para garantizar una convivencia humana en la vida social y política, una convivencia ciudadana tanto en la propia nación o Estado como en la comunidad internacional". En Latinoamérica, el concepto de ciudadanía ha cobrado vigencia social tras la crisis de la soberanía nacional. El rol de las instituciones (familia, escuela) en la formación de las virtudes y las responsabilidades ciudadanas es, entre otros, el de formar y desarrollar la facultad de imaginar, negociar, evaluar y proponer (Turbay, Amarís y Angarita, 2009).

La preocupación por los jóvenes ha dado lugar a un gran número de investigaciones que, desde distintas perspectivas, han estado orientadas a comprender las situaciones en las que viven y las diversas dimensiones que caracterizan sus formas de ser y actuar en el mundo contemporáneo (Banco Mundial, 2007; Centro Latinoamericano de Demografía (CEPAL), 2000; Escobar, Quintero, Arango y Hoyos, 2004), así como también a la formulación de políticas de juventud y a la puesta en marcha de numerosos programas dirigidos hacia ellos, muchos de los cuales proponen, explícitamente, su formación como ciudadanos (Alvarado, Fabio, Luna y Camargo, 2006; Benedicto y Morán, 2002; Henao, 2004; Rodríguez, 2003; Sarmiento, 2004).

En lo que respecta a la formación ciudadana dentro de las instituciones educativas, el trabajo de Pinilla y Torres (2006) permite evidenciar que en la escuela también se dan prácticas y procesos investigativos sobre el tema, concentrados en tres ámbitos: educación en y para los derechos humanos; resolución de conflictos y convivencia y por último, ética, educación moral y formación en valores (Henao, Ocampo, Robledo y Lozano, 2008).

García, Sales, Moliner y Ferrández (2009) investigan sobre el análisis comparado de las actitudes y los valores del profesorado universitario ante la ética profesional. Comentan que el profesorado en general está de acuerdo en que la enseñanza de los valores resulta importante y necesaria en el contexto universitario.

De igual modo, Touriñán (2009) indaga sobre el desarrollo cívico como objetivo, llevando a cabo una propuesta pedagógica. También Santos y Lorenzo (2010) investigan 
sobre la dimensión cívica en el desarrollo formativo de los estudiantes universitarios argumentando que el tema de los valores en la Universidad debe rescatarse de su uso en los discursos formalistas de las autoridades, o externos a la Universidad, para empezar en serio su exploración en el marco de una redefinición que debe desarrollar la Universidad en este siglo XXI.

En cuanto a tesis doctorales, ha habido diversos estudios sobre la educación cívica y los valores en distintas disciplinas tales como: la adquisición de valores en la enseñanza de la religión (Gual, 1996); el desarrollo moral y los valores ambientales (Nuévalos, 1997); los valores en la música en la posmodernidad (Sá Markman, 2003); pautas de transmisión de valores en el ámbito familiar (López Lorca, 2005); estudio descriptivo sobre la influencia de la sociedad de consumo en los valores y hábitos de los adolescentes de la provincia de Barcelona (Berríos, 2007) y el estudio cualitativo de las variables estructura, gestión y valores en la comunicación de las organizaciones (Morales, 2007) entre otros.

El civismo tiende a evitar las incomodidades entre las personas que se relacionan entre sí, se trata de aplicar unas pautas mínimas de comportamiento que permitan convivir en paz y libertad, respetando a las personas que nos rodean y al entorno en general. La palabra civismo tiene la misma etimología que la palabra civilización, provienen del latín civis que significa ciudadano (Segura, 2003). Así, saber ser un buen ciudadano es saber mantener una actitud civilizada. El civismo intenta hacer el trato humano lo más agradable posible a través de un comportamiento que respeta unas normas y unas costumbres sociales preestablecidas.

Hablar de civismo es hablar de ciudadanía, de un valor que significa mucho más que urbanidad. Este concepto diferencia a la persona que se interesa por su función de ciudadano comprometido y solidario en un espacio común de convivencia compartido con otros ciudadanos. De acuerdo con Ocampo, Méndez y Pavajeau (2008), la ciudadanía es más una lucha y una conquista que un punto de partida, lo cual supone, a su vez, que hacerse ciudadanos y ciudadanas es un proceso condicionado por las complejidades de los tránsitos experimentados por los individuos en su proceso de ser sujetos sociales.

La sociedad se construye gracias a ciudadanos conocedores tanto de sus derechos como de sus deberes, quienes saben armonizar su libertad personal con la responsabilidad solidaria. Los buenos modales y las normas de urbanidad son importantes; no obstante, el civismo es más que eso, no es un formalismo obligatorio, sino un sentimiento ético que permite el desarrollo de una cultura de respeto entre personas que comparten un mismo 
entorno social. Como afirman Paredes y Ribera (2006, p. 148): "mejorar el civismo es responsabilidad de todas y cada una de las personas que componemos la sociedad". Dada la importancia que tiene la educación en la formación de los ciudadanos, cabe preguntarse ¿cuál es la relevancia o el papel que desempeñan los valores en los textos de los manuales estudiados por los alumnos para su formación como futuros maestros?

De acuerdo con Prats (2011), la formación para la ciudadanía en la Universidad es una clara prioridad, según la Declaración Mundial sobre la Educación Superior en el siglo XXI de la Organización de las Naciones Unidas para la Educación, la Ciencia y la Cultura (UNESCO). Como se afirma en su artículo primero, el sistema universitario debe "contribuir a proteger y consolidar los valores de la sociedad, velando por inculcar en los jóvenes los principios en que reposa la ciudadanía democrática". En consideración con lo anterior, el objetivo de esta investigación será analizar los valores educativos en los manuales universitarios de teoría e historia de la educación utilizados en la formación de maestros, a partir de la herramienta de tipo cualitativo ATLAS.ti.

\section{Metodología}

Se trata de una investigación cualitativa de tipo descriptivo e inductivo, ya que busca conocer los fenómenos desde la perspectiva de los propios individuos (Mella, 2003). La fortaleza de la investigación cualitativa está en su dinamismo y flexibilidad para comprender los fenómenos estudiados (Cáceres, 2003); por tanto, se encuadra en un paradigma humanístico-interpretativo o constructivista. En esta investigación se ha seguido como procedimiento el análisis de contenido que supone, según Martínez (1998), una contrastación de subcategorías o primeras categorías que surgen a lo largo de un estudio en sucesivos marcos o contextos.

\subsection{Informantes clave. Muestra}

La asignatura Teorías e Instituciones Contemporáneas de Educación fue escogida tras una revisión exhaustiva de los planes de estudio vigentes:

- Resolución de 17 de febrero de 1998, de la Universidad de Murcia, por la que se hace público el plan de estudios de Maestro-Especialidad en Educación Primaria. Madrid: BOE 10/31998.

- Resolución de 11 de abril de 2002, de la Universidad de Murcia, por la que se hace público el plan de estudios de Maestro-Especialidad en Educación Primaria. 
Asimismo, se atendió a los descriptores que aparecen en la asignatura recogidos en los planes tales como: formación del ciudadano, valores cívicos, civismo, etc., al tener una conexión con nuestro problema de investigación formulado.

En cuanto a los manuales, se llevó a cabo una selección tras la visita a múltiples bibliotecas de distintas universidades de España para ver en los catálogos la cantidad de ejemplares que había publicados sobre esta asignatura. Los autores de los textos seleccionados han sido los que tenían un elevado número de ejemplares disponibles en las bibliotecas de las universidades consultadas. Aunado a esto, los autores están especializados en las áreas que nos ocupan en el estudio: magisterio, pedagogía y teoría e historia de la educación. Una vez localizado dicho material, se comprobó si en sus referencias bibliográficas los autores seleccionados se citaban entre ellos para ver la relación entre autores $y$, efectivamente, se pudo verificar que entre ellos se hacían alusión. Los autores seleccionados son los que aparecen en la tabla 1. 
Tabla 1

Autores seleccionados en la asignatura Teorías e instituciones contemporáneas de educación

Colom, A. J., Lluís, J., Domínguez, E. y Sarramona, J. (1997). Teorías e instituciones contemporáneas de educación. Barcelona: Ariel.

lyanga, A. (1998). La Educación Contemporánea. Teorías e Instituciones. Valencia: Nau Llibres.

Carreño, M. (Ed.) Colmenar, C., Egido, I. y Sanz, F. (2000). Teorías e Instituciones contemporáneas de educación. Madrid: Síntesis Educación.

García, A. (2001). Teorías e Instituciones de la educación. Una aproximación axiológica. Sevilla: Padilla Libros Editores y Libreros.

Carreño, M. (Ed.) Colmenar, C., Egido, I. y Sanz, F. (2002). Teorías e Instituciones contemporáneas de educación. Madrid: Síntesis Educación.

Colom, A. J., Lluís, J., Domínguez, E. y Sarramona, J. (2002). Teorías e instituciones contemporáneas de educación. Barcelona: Ariel.

Vicente, U. (2002). La educación: Teorías e Instituciones contemporáneas. Almería: Universidad de Almería.

Negrín, O. y Vergara, J. (2003). Teorías e Instituciones contemporáneas de educación. Madrid: Centro de Estudios Ramón Areces.

Del Pozo, Ma del Mar., Álvarez, J. L., Luengo, J. y Otero E. (2004). Teorías e Instituciones contemporáneas de educación. Madrid: Biblioteca Nueva.

Colom, A. J., Lluís, J., Domínguez, E. y Sarramona, J. (2005). Teorías e instituciones contemporáneas de educación. 4를. Barcelona: Ariel.

Negrín, O. y Vergara, J. (2006). Teorías e Instituciones contemporáneas de educación. Madrid: Centro de Estudios Ramón Areces.

Colom, A. J., Lluís, J., Domínguez, E. y Sarramona, J. (2008). Teorías e instituciones contemporáneas de educación. Barcelona: Ariel.

Carreño, M. (Ed.)., Colmenar, C., Egido, I. y Sanz, F. (2008). Teorías e Instituciones contemporáneas de educación. Madrid: Síntesis Educación.

Del Pozo, Maㅡ del M., Álvarez, J. L., Luengo, J. y Otero E. (2009). Teorías e Instituciones contemporáneas de educación. $2^{\underline{a}}$ ed. Madrid: Biblioteca Nueva.

Fuente: elaboración propia

Esta tabla 1 recoge las diferentes ediciones que los autores han publicado sobre la asignatura Teorías e Instituciones Contemporáneas de Educación, impartida, actualmente, en la titulación de Grado en Maestro de Educación Primaria en las universidades españolas. Los manuales estudiados describen objetivamente cada teoría, en la cual los autores de los manuales hacen referencia a diferentes pensadores o teóricos, e incluso, a veces, los autores aportan ideas propias en torno al tema mediante sus propias investigaciones. En cada teoría o corriente estudiada se inciden en los valores mencionados previamente, como queda recogido, a modo de síntesis, en la tabla 2. 
Tabla 2

Teorías y corrientes educativas. Valores cívicos

\begin{tabular}{|c|c|}
\hline Autores & Teorías y corrientes educativas: valores \\
\hline $\begin{array}{l}\text { Otero }(2004,2009) \\
\text { Negrín y Vergara }(2003,2006)\end{array}$ & $\begin{array}{l}\text { Teorías contemporáneas: disciplina. } \\
\text { Libertad. }\end{array}$ \\
\hline $\begin{array}{l}\text { Carreño }(2000,2002,2008) \text { y Negrín } \\
\text { y Vergara }(2003,2006) \\
\text { Del Pozo }(2004)\end{array}$ & $\begin{array}{l}\text { El movimiento de la Escuela Nueva: libertad, disciplina, } \\
\text { cooperación. } \\
\text { El movimiento de la Escuela Nueva: democracia } \\
\text { (participación activa, pensamiento colectivo de carácter } \\
\text { crítico, ciudadanía, formación del ciudadano. }\end{array}$ \\
\hline $\begin{array}{l}\text { Bernabeu }(1997,2000,2002) \text { y } \\
\text { Negrín y Vergara }(2003,2006) \\
\text { Del Pozo }(2004,2009) \text {, Negrín y } \\
\text { Vergara }(2003,2006) \text { y Carreño } \\
(2000,2002,2008)\end{array}$ & $\begin{array}{l}\text { Teorías personalistas: diálogo, relación de ayuda, } \\
\text { libertad, actitudes, desarrollo integral. } \\
\text { Teorías personalistas: democracia (justicia, igualdad, } \\
\text { diálogo, pensamiento crítico, saber escuchar a los } \\
\text { otros, tolerancia). }\end{array}$ \\
\hline
\end{tabular}

Fuente: Elaboración propia

\subsection{Procedimiento de análisis de datos}

Con el programa ATLAS.ti se han ejecutado tres actividades fundamentales: reducción de datos, disposición y transformación y finalmente, obtención y verificación de conclusiones. Cada una de estas tareas cuenta con algunas operaciones particulares, las cuales se encuentran en constante interacción durante el procedimiento analítico, tal y como lo realizan Rodríguez, Gil y García, Giménez (1999) y Pérez (1994). La búsqueda en los textos de las citas, usando categorías, es una de las herramientas más poderosas del ATLAS.ti y es muy útil cuando se posee mucho material informativo.

El poder tener a la vista, en un instante, todos los textos o citas categorizadas en un mismo rango permite captar información que sería imposible imaginar con las citas dispersas. La semiótica es una práctica especializada de lectura (Gonzalo, 1995). Son reconocidas las características de los diagramas de redes como heurísticos del conocimiento, estas permiten sintetizar información y descubrir relaciones significativas entre conceptos, facilitando su tratamiento y presentación. Por esta razón, se decidió utilizarlas en el análisis del discurso en los libros de texto estudiados.

El procedimiento seguido partió de la, ya citada, selección de los manuales estudiados en esta asignatura. En segundo lugar, el análisis partió de la revisión teórica sobre el tema objeto de estudio. En tercer lugar, se estableció un sistema de categorías para el análisis textual de los valores cívicos, particularmente, el método inductivo mediante el programa informático ATLAS.ti, versión 6.2. Finalmente, se analizaron los valores implícitos en las teorías o corrientes que aborda esta asignatura, estableciendo unos códigos o etiquetas para 
identificar el texto seleccionado. Estos códigos a su vez están dentro de una gran familia, las corrientes o teorías elaboradas en los diagramas de redes. En cada teoría o corriente estudiada se incide en los valores comentados, como queda recogido, a modo de síntesis, en la tabla 3.

Tabla 3

Valores de la educación de la ciudadanía

\begin{tabular}{|l|l|}
\hline \multicolumn{2}{|l|}{ La dignidad de la persona } \\
\hline La libertad & $\begin{array}{l}\text { - como autonomía moral } \\
\text { - como ejercicio de libertades individuales } \\
\text { - como capacidad de decisión } \\
\text { - como fundamento de derechos políticos y } \\
\text { civiles }\end{array}$ \\
\hline La justicia & $\begin{array}{l}\text { - como imparcialidad } \\
\text { - como igualdad afectiva de oportunidades }\end{array}$ \\
\hline El respeto activo & $\begin{array}{l}\text { - de las ideas y creencias } \\
\text { - de las personas } \\
\text { - de las etnias y culturas }\end{array}$ \\
\hline El civismo & $\begin{array}{l}\text { - como buenos modales } \\
\text { - normas de urbanidad } \\
\text { - diálogo como resolución civilizada de los } \\
\text { conflictos }\end{array}$ \\
\hline La responsabilidad y la participación & $\begin{array}{l}\text { - en la comunidad escolar } \\
\text { - en la comunidad social }\end{array}$ \\
\hline
\end{tabular}

Fuente: Elaboración propia

\section{Resultados: análisis y discusión}

En este artículo se han trabajado diferentes teorías: la teoría educativa en los umbrales de la contemporaneidad debido a la época que nos ocupa y a la relevancia del tema; el movimiento de la Escuela Nueva por ser un claro ejemplo para el alumnado y para los futuros formadores de magisterio. El propósito es que los discentes lo apliquen tanto en la preparación teórica de sus asignaturas como en la puesta en práctica, autónomamente, del alumnado recogido en el "plan Bolonia" — tan en boga- actualmente. Y, las teorías personalistas porque recogen una síntesis de todos los valores que deben trabajarse dentro y fuera del aula se ha llevado a cabo porque, si bien es cierto que se han trabajado más teorías dentro de cada manual, había que acotar la información retomada para esta investigación, cuyo trabajo nos ha llevado más de cinco años de estudio.

A continuación, se muestra el análisis de los resultados obtenidos en los diagramas de redes obtenidos con el programa ATLAS.ti versión 6.2, para ejemplificar cómo se han trabajado las teorías educativas. 


\section{Diagrama 1}

Teorías educativas en los umbrales de la contemporaneidad

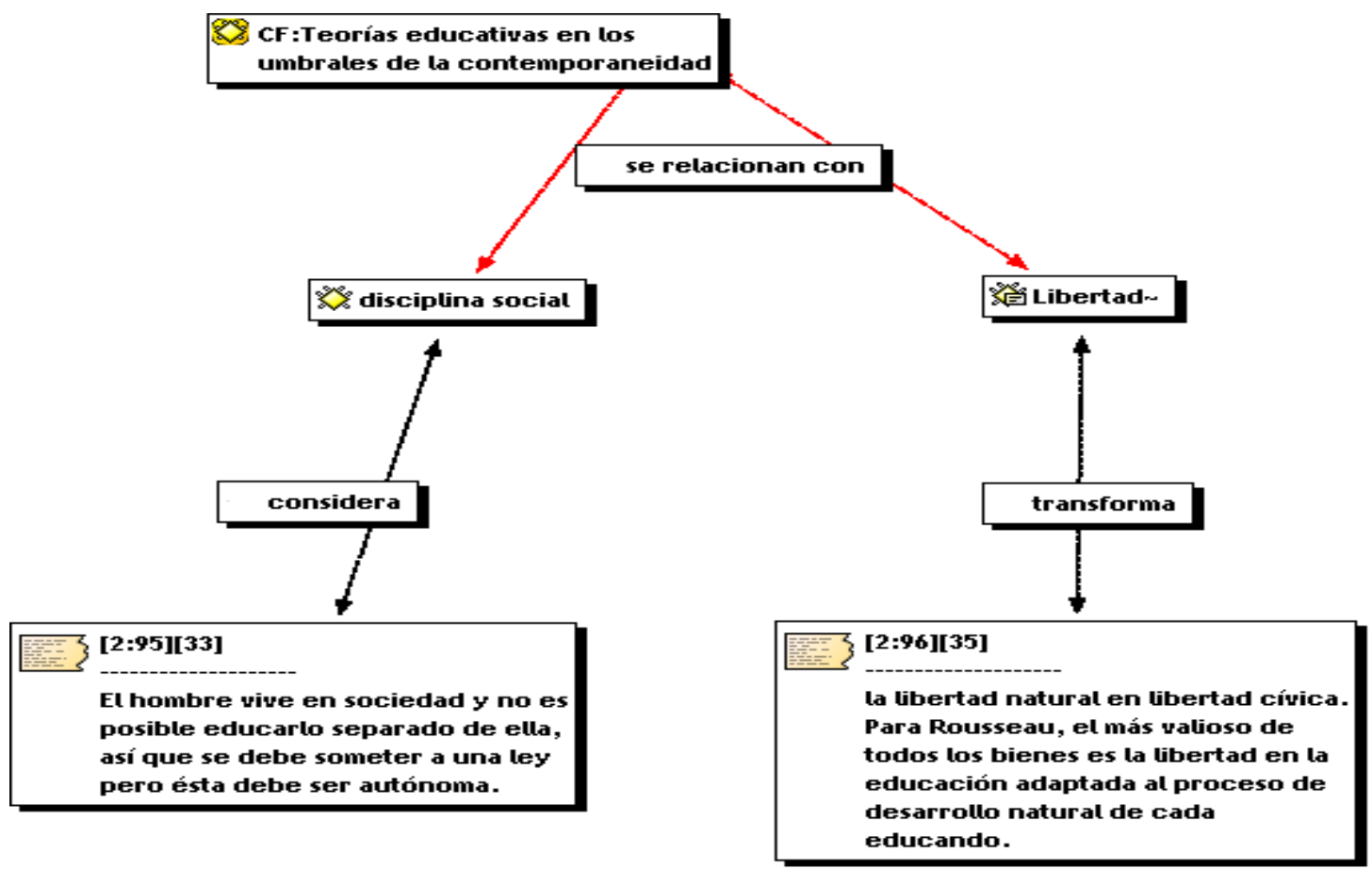

Fuente: Elaboración propia

En el diagrama 1, se tienen muy en cuenta la libertad, la disciplina, la responsabilidad, el sentido del deber, la moralidad, la conciencia moral, la autonomía, el sentido crítico, el conocimiento, la experiencia, la educación, la formación y el alumnado, y el saber práctico.

lyanga (1998) señala que el origen de estas teorías está localizado en la crisis de valores. Según este autor, hay valores que casi han desaparecido y otros que han sido tergiversados como los religiosos, los cívicos, los afectivos, los sociales y los estéticos. La carencia de unos valores a los que aferrarse, y que sirvan de guía a la vida -explica lyanga - deja a ésta sin rumbo, contribuyendo a la desorientación y a la inseguridad vital. La preocupación por el futuro, siempre presente en el hombre, ha ido creciendo progresivamente en el siglo XX. De ahí que muchos autores aborden este tema, como es el caso de Spranger, Böhler, Entralgo y Cariacedo, entre otros. Spranger (citado en lyanga, 1998, p. 80) explica tres actitudes del hombre frente al futuro:

La necesidad del hombre frente a los acontecimientos futuros regidos por las leyes naturales, y la esfera de la necesidad que se circunscribe con nombres como destino, 
fatalidad y causalidad. El querer de nuestra voluntad, que además de respetar las leyes naturales influye en ellas por la técnica. Y el deber o situación de la conciencia moral.

lyanga (1998) sostiene que la educación consiste más, que en adquirir información o saberes concretos, en aprender a aprender y en desarrollar actitudes abiertas y flexibles, adquiriendo firmeza para saber tomar decisiones y capacidad de comunicación.

Rousseau (citado en Negrín y Vergara, 2003 y Del Pozo, 2004), en su obra El Contrato Social, hace referencia al concepto de libertad cívica. La naturaleza es un referente educativo prioritario en la pedagogía de Rousseau, quien se manifiesta contra la educación verbalista y libresca y plantea una nueva pedagogía y una nueva filosofía de la educación basadas en los intereses y necesidades del alumno/a y en el desarrollo natural en libertad. Mediante el contrato —explica Rousseau— los hombres dispersos entregan, recíprocamente, su libertad natural en un pacto de asociación realizado entre iguales, y que da lugar a la persona moral. Esto no significa que las personas deban renunciar a sus intereses en razón del bien común, sino que la ley es la expresión de la propia voluntad ética. Es decir, que las personas actúan más por convicciones cívicas que por coerciones externas.

En las teorías educativas en los umbrales de la contemporaneidad, los alumnos son el centro de interés. El saber práctico es importante pero tanto, o más, lo es el saber teórico fundamentado en diferentes disciplinas para así poder formar al alumnado en todos los sentidos y desarrollar todas sus potencialidades. Locke (citado en Del Pozo, 2004, p. 177) afirma que:

La tarea propia del hombre es la de buscar la felicidad y evitar la miseria. La felicidad es lo que contempla y deleita el espíritu, la miseria lo que lo perturba, descompone o atormenta. Los placeres que él considera más duraderos de esta vida son la salud, la reputación, el conocimiento, hacer el bien y la esperanza de una felicidad eterna. 


\section{Diagrama 2. El movimiento de la Escuela Nueva}

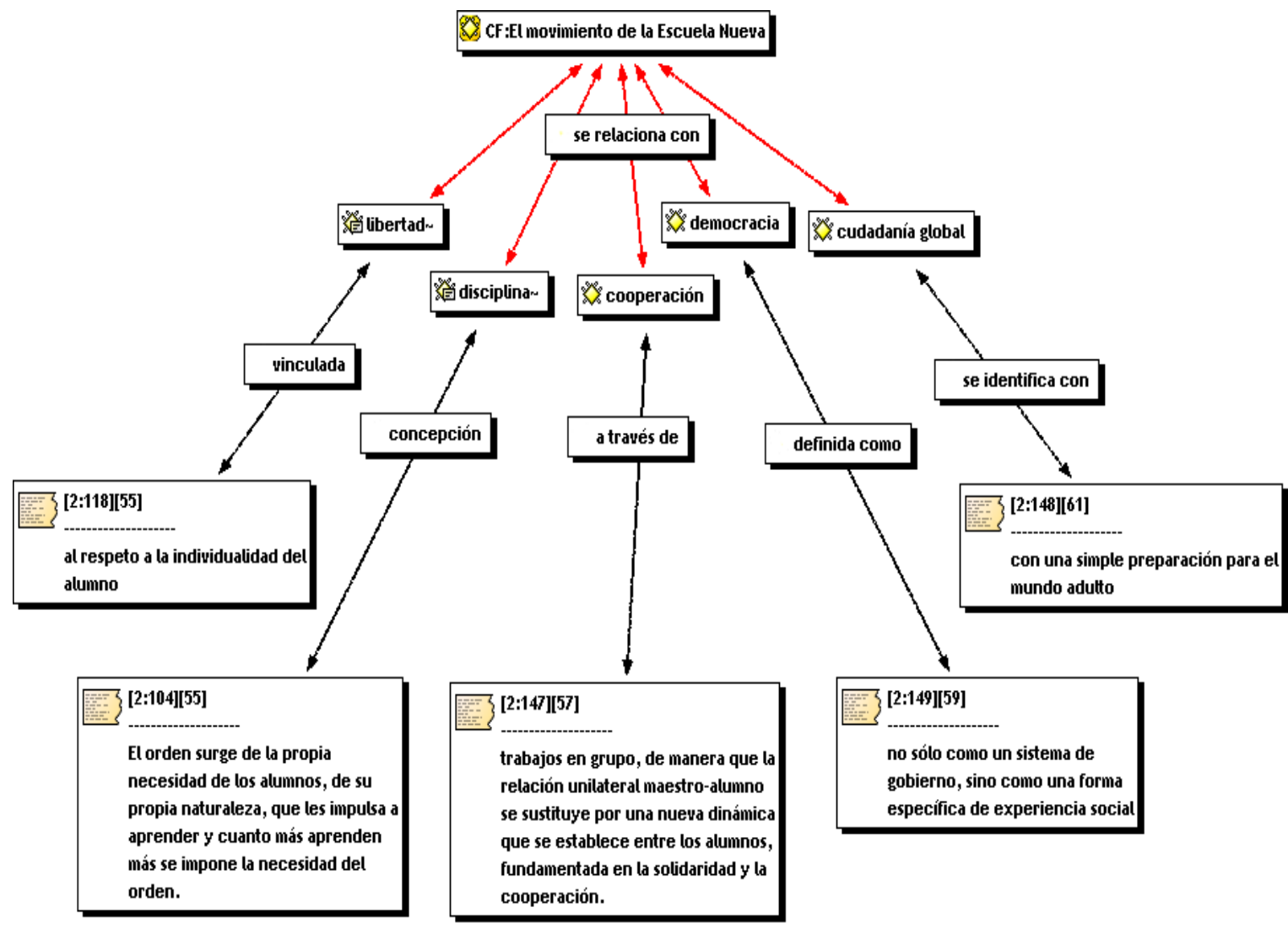

Fuente: Elaboración propia

En el diagrama 2, la finalidad educativa de preparar para la vida, tan característica de la Escuela Nueva, se empezó a entender como una mirada hacia el futuro con propósitos transformadores, como una forma de reconstruir la sociedad a través de la educación, lo cual implicaba preparar al potencial ciudadano en la comunidad y, aún más, prepararle para una ciudadanía global, para ser parte de la humanidad. En las teorías y los métodos de la Escuela Nueva, la escuela debe ser activa para poder girar en torno a los intereses del alumnado y para que la enseñanza y el aprendizaje sean más eficaces. Los precursores de este movimiento son Rousseau, Pestalozzi, los principios de Tolstoi, Key, Fröbel y Herbart (citado en Vicente, 2002 y Negrín y Vergara, 2003).

Luzuriaga (citado en Marín, 1988, p. 261) en la Educación Nueva establece una distinción fundamental "entre las corrientes pedagógicas actuales, una de las que más interés ofrecen, por su originalidad y difusión, es la que, sintéticamente, se denomina 
educación nueva". Marín (1988) explica que la Escuela Nueva intenta preparar al alumnado para la vida moderna con sus exigencias materiales y cívicas. La Escuela Nueva educa la conciencia moral, ofreciendo cada tarde al alumnado, lecturas, relatos tomados de la vida ficticia o real y provocando en ellos reacciones espontáneas, verdaderos juicios de valor, que fortalecen su conciencia y los determinan al bien. Los profesores se liberan así de toda la parte disciplinar y pueden consagrarse enteramente al progreso intelectual y moral de los alumnos.

El valor de la libertad se centra en respetar la individualidad del alumnado y la cooperación entre ellos. Se trata de no desarrollar al individuo en actividades aisladas, sino mediante trabajos en común con los demás y al servicio de los otros. Son elementos fundamentales: el espíritu crítico, la formación y la conciencia moral, la responsabilidad, la autonomía personal, la educación moral, la educación ciudadana y democrática, la preparación ciudadana y el desarrollo de caracteres religiosos-cívicos.

En la escuela debe vivirse en comunidad y de manera solidaria, no puede concebirse como una reunión de individuos aislados, sino como una verdadera sociedad en la que, incluso, llegue a eliminarse el sentido de rivalidad, este debe sustituirse por el de compañerismo y solidaridad. Se debe respetar al alumnado en sus actitudes y capacidades para que él mismo desde adentro pueda desarrollar lo mejor de sí y ponerse en una situación dinámica de aprendizaje y de responsabilidad.

A través de actividades escolares realizadas en grupos o equipos se desarrollan hábitos positivos de convivencia y cooperación social en el alumnado, preparándoles para la vida. Moore (citado en García Benítez, 2001) comenta que el movimiento de la Escuela Nueva, dentro de los modelos de educación, se considera un enfoque progresivo u orientado al alumno, el cual le anima a tomar la iniciativa a diferencia del enfoque tradicional donde la enseñanza daba órdenes, coaccionaba y prohibía.

Ferrière (citado en Marín, 1988) fue el principal responsable de la creación de la Oficina Internacional de las Escuelas Nuevas. Este autor (citado en Negrín y Vergara, 2003, p. 36) afirma que la Escuela Nueva es "ante todo un internado familiar situado en el campo, donde la experiencia personal del niño es el fundamento tanto de la educación intelectual, en particular por el recurso de los trabajos manuales, como de la educación moral mediante la práctica del sistema de la autonomía relativa de los alumnos".

Demolins (citado en Vicente, 2002 y Negrín y Vergara, 2003) funda Ecole des Roches. El objetivo principal de esta institución era formar la personalidad moral, inspirar la iniciativa y 
el sentido de la responsabilidad. En 1906, Lietz (citado en Negrín y Vergara, 2003) fundó una comunidad libre en Turingia junto a sus dos colaboradores Wyneken y Geheeb, donde prestaba especial énfasis en la coeducación e incluía en su programa el desarrollo de caracteres religiosos-cívicos.

Del Pozo (2004) argumenta que, si la escuela tradicional basa su eficacia en la competitividad, en la emulación y en los sistemas de recompensas y castigos, la Escuela Nueva fomenta la cooperación entre los alumnos y estimula el espíritu comunitario. Finalmente, si la escuela tradicional adolece de una absoluta falta de libertad, la Escuela Nueva defiende la autonomía de los escolares como paso previo para la autorresponsabilidad personal. Según la autora, el elemento central del pensamiento pedagógico de la Escuela Nueva, en sus primeros momentos, era el paidocentrismo, esto es, la enseñanza centrada en el alumno, que configuró una nueva relación educador-educando.

Esta enseñanza desemboca, en último término, en un antropocentrismo, en la búsqueda del hombre completo que se conseguirá a través de la educación. Del Pozo (2004) comenta que el ideal educativo de la Escuela Nueva es la educación integral, en la que, además del cultivo tradicional del intelecto, se toman en cuenta los aspectos físicos, sociales, estéticos y emocionales del individuo. En el Movimiento de la Escuela Nueva se considera que el alumnado es el protagonista principal del proceso de enseñanza. Se intenta que se forme a través de su experiencia e instintos naturales —como sostenía Rousseaudesarrollando valores de participación, responsabilidad, autonomía personal, justicia y libertad para actuar y libertad de pensamiento.

Se debe incidir en la formación del alumnado y en su conciencia moral, atendiendo a las necesidades de la comunidad. Desde nuestro punto de vista, estas características son necesarias y esenciales para un buen desarrollo del aprendizaje pero resulta fundamental que el aprendizaje y la adquisición de conocimientos no sólo deriven de la experiencia del sujeto, sino que haya una instrucción académica, unos contenidos distribuidos en diferentes materias para que el alumnado adquiera un bagaje cultural diverso. Es importante, además, que logre una maduración académica y personal que le permita mostrar el saber aprendido a través de la realización de pruebas y exámenes.

Es necesario que exista un deber por parte del docente de enseñar, instruir, corregir, guiar la enseñanza y el aprendizaje con una disciplina moderada. Y, por otro lado, que el discente debe estudiar los conocimientos adquiridos, aprender por ensayo-error las habilidades que va ejerciendo y demostrar sus destrezas, capacidades y actitudes tanto entre 
sus compañeros como con el profesorado en cuestión. También es necesario que esta escuela se lleve a cabo no solamente al aire libre - trabajo de campo, excursiones, salidas, etc.- sino que es necesario que el alumnado sepa convivir y formarse tanto dentro como fuera de una institución académica. Sí que es necesaria la práctica del día a día y es la mejor forma para aprender viendo la realidad y experimentándola, así como también, deben de trabajar y aprender a educarse dentro de la institución.

Diagrama 3. Teoría personalista

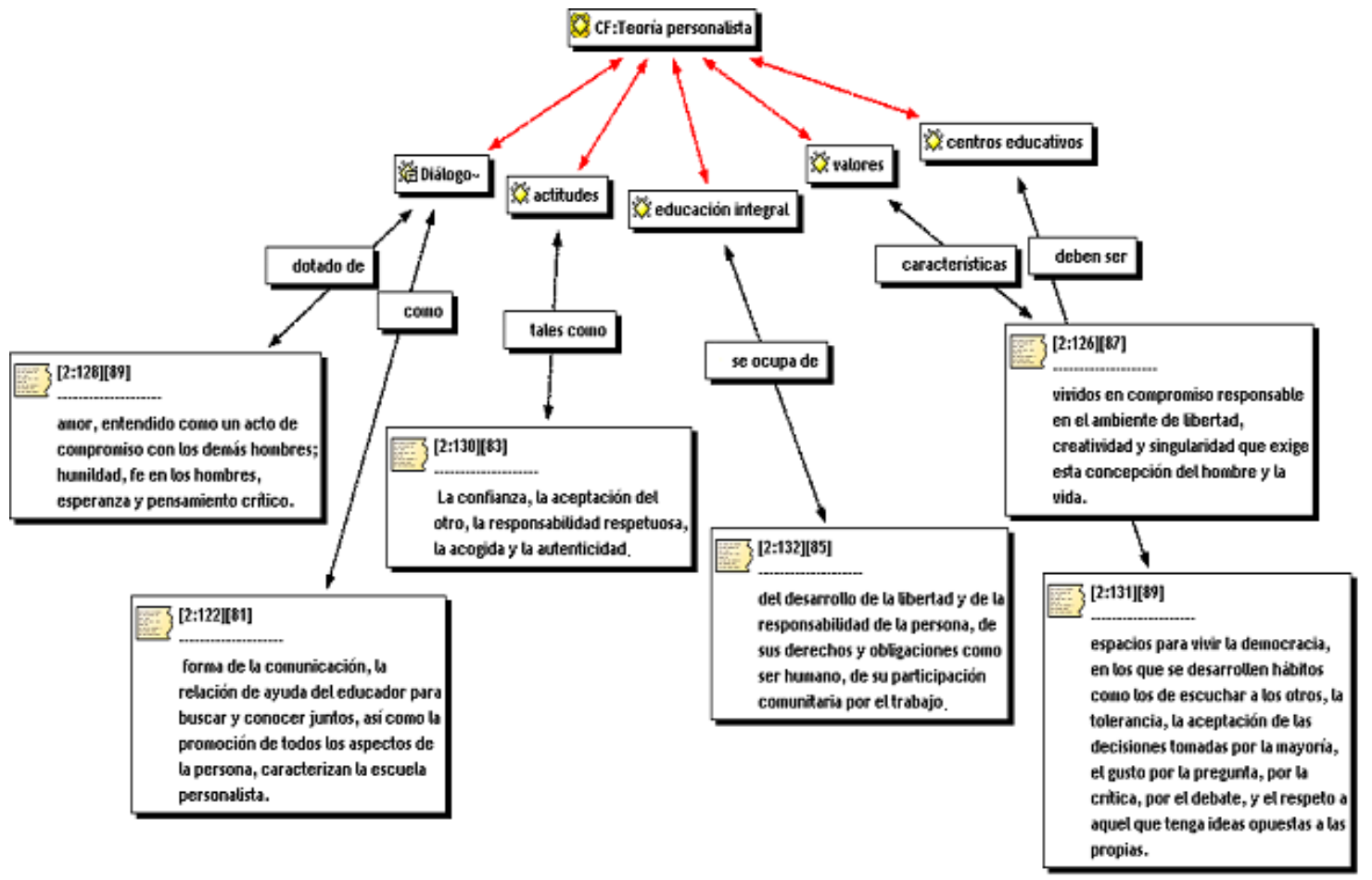

Fuente: Elaboración propia

En el diagrama 3, en la teoría personalista, la relación educativa entre el educador y el educando supone una comunicación que se manifiesta en el diálogo y alcanza los planos afectivo e intelectual. La consideración del educando como persona incluye su vocación perfectiva, sobre todo en la libertad, responsabilidad, capacidad interrogativa y de iniciativa para crear algo original, cooperación, formación en valores, así como su apertura a los demás y hacia una existencia transformadora. Los rasgos de una escuela personalista actual vendrían dados por su carácter comunitario, de apertura a la sociedad, por ser activa y crítica, por promover la participación democrática y por proseguir como objetivo de la educación el desarrollo integral de la persona humana. 
Scheller y Mounier (citado en Colom, 1997, p. 127) destacan como soporte principal de la persona "los valores cívicos, la confianza, la aceptación al otro, la acogida y la autenticidad como las nuevas actitudes que regulan la relación educativa personalista”. Un ejemplo de ello - explican estos autores - es la escuela de Barbiana, lugar que fomentaba las actitudes comunitarias en el uso de sus materiales y en sus actividades. En este sitio los problemas comunes eran abordados desde una concepción de justicia apoyando a los más débiles.

También Mounier (citado en Negrín y Vergara, 2003 y Del Pozo, 2004), en su Manifiesto al servicio del personalismo, entiende a la persona como un ser espiritual dotado de una escala de valores libremente adoptada. Aquí aparecen reunidas dos de las características más importantes de esta corriente; por un lado, la creencia en la libertad del ser humano,; y, por otro lado, la responsabilidad para comprometerse con esos valores y hacerlos el eje de su trayectoria vital.

Maritain (citado en lyanga, 1998, p. 71) centra estas teorías en la persona reivindicando su valor y dignidad. Manifiesta que "la educación integral debe contemplar la educación y su proceso, la educación moral y política, las reglas de actuación y la reintegración de las masas". De igual modo, Maritain (citado en Negrín y Vergara, 2003), en su obra Humanismo integral, proponía la búsqueda de una civilización personalista y comunitaria fundada sobre los derechos humanos, la cual diera satisfacción a las aspiraciones y necesidades sociales del hombre, este autor afirma que sólo la educación integral del individuo es personalista.

Del Pozo (2004) considera que el personalismo concedió una gran importancia a la educación. Los personalistas consideraron que las dos finalidades más importantes de la educación son las de transmitir valores y preparar al ser humano para el compromiso. De ahí, el papel fundamental que concedieron a la figura del profesor, encargado de mostrar a los educandos esos valores y formarles en el compromiso vital. Freire (citado en Negrín y Vergara, 2003 y Del Pozo, 2004) sostiene que los centros educativos deben convertirse en espacios para vivir la democracia.

Se coincide con las teorías personalistas en la importancia concedida a la formación de valores, en que son necesarios y esenciales en la vida de una persona para sentirse plena en la sociedad en la que vive; y también, para poder participar como ciudadanos en la comunidad en la que forman parte habiendo adquirido una formación integral y completa a nivel intelectual, personal, social, moral y cívico.

El papel atribuido al educador personalista difiere del tradicional, pues se funda en la libertad, la transmisión del conocimiento cede paso a la relación de ayuda para buscar y 
conocer juntos. La confianza, la aceptación del otro, la responsabilidad respetuosa, la acogida y la autenticidad son las nuevas actitudes que regulan la relación educativa personalista; las tareas y su ritmo se adaptan a las aptitudes e intereses diferenciales de los educandos, es su dignidad personal la que define la praxis escolar.

\section{Conclusiones}

Esta investigación se ha planteado debido a la escasez de estudios sobre los valores en los manuales universitarios para la formación de los maestros en la Universidad. En España, como ocurre, por ejemplo en Chile (Alarcón, Díaz, Tagle, Ramos y Quintana, 2014), los educadores han de trabajar medidas de fortalecimiento para la formación inicial y el desarrollo profesional docente-discente. Sánchez (2009) hace hincapié en los docentes como profesionales que forman parte del dispositivo por medio del cual la sociedad intenta preparar para la vida adulta a sus nuevas generaciones.

Es importante difundir estos conocimientos a los diferentes investigadores para apostar por una educación en valores más acorde con el mundo actual tanto para las jóvenes generaciones como para los futuros maestros.

La utilización de la herramienta ATLAS.ti es un algo novedoso y poco utilizado para trabajar la metodología cualitativa. Asimismo, este programa resulta eficaz para la elaboración y representación de redes semánticas de contenido en el análisis textual.

Los resultados de nuestra investigación coinciden con los hallazgos de Morales (2014), en los cuales se hace manifiesto que hay que fomentar el pensamiento crítico en el alumnado mediante el trabajo en el aula. Para ello, se pueden trabajar las teorías contemporáneas como un marco analítico y teórico explicativo, aplicable en diferentes disciplinas, tales como: las ciencias sociales, la pedagogía y la teoría y la historia de la educación, aplicando la teoría aprendida a la práctica escolar y así adquirir un buen bagaje profesional en la tarea docente.

A la pregunta que nos hacíamos en el artículo sobre ¿cuál es la relevancia de los valores en los textos de los manuales de teoría e historia estudiados por los alumnos para su formación como futuros maestros? Debemos considerar que actualmente, los valores son cualidades adquiridas no innatas, que les posibilitan a las personas ser más tolerantes, comprender diferentes puntos de vista, analizar y reflexionar nuestro comportamiento y el de los demás a través de la libertad, la responsabilidad, la participación y la empatía, entre otros valores. 
Tanto en los manuales de teoría e historia de la educación como en los demás manuales universitarios utilizados con nuestro alumnado en clase, destacamos teóricamente los valores que se les intenta transmitir, por ejemplo, con una imagen, con un símbolo, con palabras, con diferentes textos, etc. Los docentes podemos potenciar, en el estudiante, diferentes maneras de comprender el mundo que le rodea y fomentar actitudes positivas hacia sí mismo, con los demás y en la sociedad en la que está inmerso.

Aconsejamos, desde nuestro punto de vista como profesores en ejercicio, que en la práctica docente, los valores analizados en este artículo deben trabajarse a fondo y llevarse a cabo. Consideramos que la libertad es necesaria tanto dentro como fuera del aula, una libertad dirigida pero no impuesta porque mediante ella el estudiante se siente más libre para razonar, exponer sus argumentos en clase con los compañeros, preguntar sus dudas y cuestionar sus diferentes puntos de vista en relación con las asignaturas trabajadas. Ser orientadores, guías del proceso de aprendizaje de nuestros estudiantes, sin mostrar severidad, con un acercamiento de feedback, una bidireccionalidad profesor-estudiante.

La justicia debe ejercitarse en un plano de igualdad, la concebimos así en el marco educativo, igualdad de derechos en el alumnado, de trato, en el cumplimiento de deberes, etc., evitando la exclusión, la xenofobia, la marginación, el bullying, y para eso debemos trabajar mediante ejercicios prácticos, a través del diálogo y de diferentes prácticas educativas, vídeos, representaciones teatrales, aprendizaje basado en problemas, centros de interés, talleres, etc.

El respeto activo es fundamental entre los profesores y los alumnos. Saber escucharse entre todos, respetar los pensamientos, la iniciativa del estudiante y valorar que cada persona es diferente y que, como tal, tiene derecho a ser respetada en su forma de ser y de actuar. Hay que potenciar la discusión y el diálogo en clase para fomentar la expresión y el pensamiento crítico tan necesario para la formación de nuestros estudiantes como futuros maestros.

Asimismo, resulta esencial incidir en la responsabilidad y la participación, no sólo para quienes se vayan a dedicar a la docencia, sino que en cualquier profesión es necesario adquirir estas destrezas y valores para desempeñarlos en la sociedad. Ejercitar la responsabilidad en el cumplimiento de tareas, en llevarlas a término y comprometerse con la realización de labores participando activamente tanto en el ámbito escolar como fuera del mismo. 
El civismo es un valor que recoge, desde nuestro punto de vista, todos los valores que conforman al ser humano, que forman a la persona. Debemos trabajarlo intensamente con propuestas didácticas útiles y eficaces para la práctica en ejercicio de la profesión.

Hemos destacado, en este artículo, tres teorías representativas. En primer lugar, las teorías educativas en los umbrales de la contemporaneidad, que precisamente subrayan estos valores y que acentúan su potenciación. En segundo lugar, el movimiento de la Escuela Nueva, el cual aboga por la creatividad del alumnado y por fomentar una enseñanza dirigida pero libre, sin restricciones, abierta al estudiante, dirigida a él, a sus intereses y demandas. Por último, la teoría personalista centrada en la educación y en los centros educativos como vías fundamentales para formar a los educandos.

Si nos fijamos en estas teorías unidas, las tres constituyen un ejemplo a seguir en nuestro día a día con nuestro alumnado, porque se trata grosso modo de que nuestros jóvenes no pierdan el norte y sepan el camino a seguir, orientándolos del mejor modo, trabajando con ellos con libertad y disciplina pero con respeto e igualdad (justicia), para finalmente, poder formarse como unos ciudadanos que puedan ejercer su práctica profesional con un verdadero código ontológico, participando activamente en la sociedad como ciudadanos democráticos.

Estas teorías educativas son una muestra representativa para indicar los puntos a seguir en la práctica para que los docentes formen a los futuros maestros, aunque hemos abarcado muchas más teorías y manuales en nuestra investigación. Hacemos constatar que, como explicamos anteriormente, hay diferentes tipos de valores y se pueden trabajar de distinta manera, nosotros consideramos fundamental que, a través de la historia, que es el origen de nuestra existencia y de nuestra vida, debemos acercar las diferentes cosmovisiones y creencias, y la relación con el contexto social dentro y fuera del aula.

Se ha llevado a cabo una investigación sincrónica para analizar, en la época actual, los valores en auge, especialmente, los valores cívicos y con ello concretar qué es lo que aprenden los futuros maestros en esta asignatura de Teorías e Instituciones Contemporáneas de Educación. En futuras investigaciones se tratará de dirigirse hacia el pasado, es decir, hacer ver la evolución diacrónica existente en los manuales para concretar y argumentar sobre qué valores se incide más en el pasado, en concreto en las etapas del franquismo, la transición y la democracia. 
De igual modo, nos plantemos que se debe analizar lo ocurrido en el aula con estos profesores, hecho que llevaremos a cabo en las próximas investigaciones, Este se puede realizar mediante estudios etnográficos o estudios de casos. Investigaciones de este tipo pueden darnos más señas de identidad sobre cómo se utilizan los valores en clase para enseñar las diferentes épocas históricas, además, podríamos conocer los tipos de interacción que propician los docentes al hablar de temas tan necesarios como los valores cívicos.

\section{Referencias}

Alarcón, Paola, Díaz, Claudio, Alarcón Tagle, Tania, Ramos, Lucía y Quintana, María. (2014). Metáforas para profesor y estudiante de pedagogía en un grupo de estudiantes de pedagogía chilenos. Actualidades Investigativas en Educación, 14(2), 1-31. DOI: http://dx.doi.org/10.15517/aie.v14i2.14829

Alvarado, Sara Victoria, Fabio, Héctor, Luna, María Teresa y Camargo, Marina. (2006). Transformación de actitudes frente a la equidad en niños y niñas de sectores de alta conflictividad social, en un proceso de socialización política y educación para la paz. Revista Latinoamericana de Ciencias Sociales. Niñez y Juventud, 4(1), 217-250.

Banco Mundial. (2007). Informe sobre el desarrollo mundial, 2007. El desarrollo y la próxima generación. Panorama general. Washington DC.: Banco Internacional de Reconstrucción y Fomento /Banco Mundial.

Benedicto, Jorge y Morán, María Luz. (2002). La construcción de una ciudadanía activa entre los jóvenes. Madrid: Instituto de la Juventud.

Berríos, Llareda Alejandra. (2007). La influencia de la sociedad de consumo en los valores y hábitos de los adolescentes de la provincia de Barcelona. (Tesis doctoral). Universidad de Barcelona, Barcelona, España.

Cáceres, Pablo. (2003). Análisis Cualitativo de Contenido: una alternativa metodológica alcanzable. Psicoperspectivas, 2(1), 53-82.

Carreño, Miryam (Ed.), Colmenar, Carmen, Egido, Inmaculada y Sanz, Florentino. (2000). Teorías e Instituciones contemporáneas de educación. Madrid: Síntesis Educación.

Carreño, Miryam (Ed.), Colmenar, Carmen, Egido, Inmaculada y Sanz, Florentino. (2002). Teorías e Instituciones contemporáneas de educación. Madrid: Síntesis Educación.

Carreño, Miryam (Ed.), Colmenar, Carmen., Egido, Inmaculada y Sanz, Florentino. (2008). Teorías e Instituciones contemporáneas de educación. Madrid: Síntesis Educación.

Centro Latinoamericano de Demografía (CEPAL). (2000). Juventud, población y desarrollo en América Latina y el Caribe. Problemas, oportunidades y desafíos. Santiago de 
Chile: Comisión Económica para América Latina. Fondo de Población de las Naciones Unidas - NUAP.

Cifuentes, Luis María. (2008). La educación y los valores cívicos. Revista de la Asociación de Inspectores de Educación de España, 9, 1-6.

Colom, Antoni, Bernabeu, Josep Lluís, Domínguez, Emilia y Sarramona, Jaume. (1997). Teorías e instituciones contemporáneas de educación. Barcelona: Ariel.

Colom, Antoni, Bernabeu, Josep Lluís, Domínguez, Emilia y Sarramona, Jaume. (2002). Teorías e instituciones contemporáneas de educación. Barcelona: Ariel.

Del Pozo, María del Mar, Álvarez, José Luis, Luengo, Julián y Otero, Eugenio. (2004). Teorías e Instituciones contemporáneas de educación. Madrid: Biblioteca Nueva.

Del Pozo, María del Mar, Álvarez, José Luis, Luengo, Julián y Otero, Eugenio. (2009). Teorías e Instituciones contemporáneas de educación ( $2^{\underline{a}}$ ed.). Madrid: Biblioteca Nueva.

Escámez, Juan. (2002). La educación de la ciudadanía. Madrid: Editorial CCS.

Escobar, Manuel Roberto, Quintero, Fernando, Arango, Ana María y Hoyos, Diana. (2004). Estado del arte del conocimiento producido sobre jóvenes 1985-2003. Bogotá: Programa Presidencial Colombia Joven. Agencia de Cooperación Alemana GTZ, UNICEF Colombia. Recuperado de https://semillerojovenes.files.wordpress.com/2010/07/informe-estado-del-arte-sobrejovenes-1985-2003.pdf

García Benítez, Antonio. (2001). Teorías e Instituciones de la educación. Una aproximación axiológica. Sevilla: Padilla Libros Editores y Libreros.

García López, Rafaela, Sales, Auxiliadora, Moliner, Odet y Ferrández, Reina. (2009). La formación ética profesional desde la perspectiva del profesorado universitario. Teoría de la educación, 21(1), 199-221.

Gervilla, Enrique. (1992). Los valores y cultura de los jóvenes. Madrid: Dikynson.

Gonzalo, Abril. (1995). Análisis Semiótico del Discurso. En Juan Manuel Delgado y Juan Gutiérrez (Coords.), Métodos y Técnicas Cualitativas de Investigación en Ciencias Sociales (pp. 427-463). Madrid: Síntesis.

Gual, Alfonso. (1996). La adquisición de valores en la enseñanza de la religión. (Tesis doctoral). Universidad Jaume I, Castellón, España.

Henao, Juanita. (2004). La formulación de la política de juventud de Bogotá: un ejercicio de democracia participativa y de construcción de futuro. Revista Latinoamericana de Ciencias Sociales, Niñez y Juventud, 2(2), 108-144.

Henao, Juanita, Ocampo, Angélica María, Robledo, Ángela María y Lozano, Martha Cecilia. (2008). Los grupos juveniles universitarios y la formación ciudadana. Universitas Psychologica, 7(3), 853-867. 
lyanga, Augusto. (1998). La Educación Contemporánea. Teorías e Instituciones. Valencia: Nau Llibres.

López Lorca, Hortensia. (2005). Pautas de transmisión de valores en el ámbito familiar. Tesis doctoral. Universidad de Murcia.

Marín, Ricardo. (1988). Principios de la educación contemporánea. Madrid: Rialp.

Martínez, Manuel. (1998). La investigación cualitativa etnográfica en educación. México: Trillas.

Mella, Orlando. (2003). Metodología cualitativa en ciencias sociales y educación: orientaciones teórico-metodológicas y técnicas de investigación. Santiago: Primus.

Morales, Francisca. (2007). Estudio cualitativo de las variables estructura, gestión y valores en la comunicación de las organizaciones. (Tesis doctoral). Universidad Autónoma de Barcelona, Barcelona, España.

Morales, Luis Carlos. (2014). El pensamiento crítico en la teoría contemporánea. Actualidades investigativas en educación, 14 (2), 1-23. DOI: http://dx.doi.org/10.15517/aie.v14i2.14833

Negrín, Olegario y Vergara, Francisco Javier. (2003). Teorías e Instituciones contemporáneas de educación. Madrid: Centro de Estudios Ramón Areces.

Negrín, Olegario y Vergara, Francisco Javier. (2006). Teorías e Instituciones contemporáneas de educación. Madrid: Centro de Estudios Ramón Areces.

Nuévalos, Carmen Pilar. (1997). El desarrollo moral y los valores ambientales. (Tesis doctoral). Universidad de Valencia, Valencia, España.

Ocampo, Angélica María, Méndez, Sara y Pavajeau, Carol. (2008). Las subjetividades como centro de la formación ciudadana. Universitas Psychologica, 7(3), 837-851.

Paredes, Eulalia y Ribera, Dolores. (2006). Educar en valores. Barcelona: Tibidabo.

Pérez, Gloria. (1994). Investigación Cualitativa: Retos e interrogantes. Madrid: La Muralla.

Pinilla, Alexis Vladimir y Torres, Juan Carlos. (2006). De la educación para la democracia a la formación ciudadana: una década de incertidumbres. Bogotá: Universidad Pedagógica Nacional / Instituto para el Desarrollo Pedagógico y la Investigación Educativa IPEP.

Prats, Joaquín. (10 de marzo, 2011). Educación para la ciudadanía en la Universidad. Escuela, Núm. 3897 (387), p. 3.

Pujol, Esteve y Luz, Inés. (2003). Valores para la convivencia. Barcelona: Parramón.

Rodríguez, Gregorio, Gil, Javier y García Jiménez, Eduardo. (1996). Metodología de la investigación cualitativa. Archidona, Málaga: Ediciones Aljibe. 
Rodríguez, Ernesto. (2003). Políticas públicas de juventud en América Latina: de la construcción de espacios específicos al desarrollo de una perspectiva generacional. Revista Latinoamericana de Ciencias Sociales, Niñez y Juventud, 1(2), 15-49.

Sá Markman, Rejane. (2003). Los valores en la música en la posmodernidad. (Tesis doctoral). Universidad Autónoma de Barcelona, Barcelona, España.

Sánchez, Christian. (2009). Formación de profesores en Educación Básica en Chile: el desafío del dominio disciplinar y pedagógico. Perspectivas educacionales, (10), 1-11.

Santos, Miguel Ángel y Lorenzo, María del Mar. (2010). Dimensión cívica y desarrollo formativo de los estudiantes universitarios en el contexto español. Revista electrónica de investigación educativa, 12(1), 1-17.

Sarmiento, Libardo. (2004). Política Pública de Juventud en Colombia. En Germán Muñoz (Ed.), Construcción de Políticas de Juventud (pp. 115-193). Manizales, Colombia: Universidad de Manizales.

Segura, Santiago. (2003). Nuevo Diccionario etimológico Latín - Español y de las voces derivadas. Bilbao: Universidad de Deusto.

Turbay, Marco Antonio, Amarís, María y Angarita, Consuelo. (2009). Estudio de caso acerca del significado de la formación ciudadana y su relación con las múltiples inteligencias en el ser humano: una aproximación desde la institución familiar y educativa en la ciudad de Barranquilla, Colombia. Scientific Journals, 8(1), 161-174.

Touriñán, José Manuel. (2009). El desarrollo cívico como objetivo. Una propuesta pedagógica. Teoría de la educación, 21(1), 129-159.

Vicente, Ulpiano. (2002). La educación: Teorías e Instituciones contemporáneas. Almería: Universidad de Almería. 